\title{
Eficácia da técnica de relaxamento com imagem guiada em pacientes oncológicos submetidos a tratamento quimioterápico
}

\author{
Efficacy of guided imagery relaxation in chemotherapy-treated cancer patients
}

\author{
Claudia Adriana Facco Lufiego ${ }^{1} \bowtie$, Rodolfo Herberto Schneider'1, Ângelo José Gonçalves Bós ${ }^{1}$ \\ ${ }^{1}$ Instituto de Geriatria e Gerontologia da Pontifícia Universidade Católica do Rio Grande do Sul (IGG-PUCRS). Porto Alegre, RS.
}

\section{RESUMO}

Objetivos: avaliar a eficácia da técnica de relaxamento com imagem guiada em pacientes oncológicos submetidos a tratamento quimioterápico em relação à diminuição dos níveis de depressão e ansiedade.

Métodos: Estudo do tipo ensaio clínico não randomizado. A amostra foi constituída de participantes de ambos os sexos, com idade igual ou superior a 30 anos e que estavam iniciando tratamento quimioterápico em um hospital universitário de Porto Alegre. Os pacientes foram divididos em dois grupos: Grupo Experimental, no qual foi aplicada a técnica de relaxamento, e Grupo Controle, sem a intervenção. As sessões de relaxamento com a técnica de imagem guiada tiveram duração de 12 minutos e foram realizadas durante um período de 14 semanas, sempre durante o procedimento quimioterápico. Para avaliar os níveis de depressão e ansiedade foram aplicados os instrumentos Inventário de Depressão de Beck e Inventário de Ansiedade de Beck no período inicial e final do estudo. Os dados foram analisados no software estatístico Epi Info 7.0, utilizando os testes t de Student, Wilcoxon-Mann-Whitney e Qui-quadrado de Pearson. Foram considerados significativos valores de $\mathrm{p}$ inferiores a 0,05 .

Resultados: Foram incluídos 113 participantes, com média de idade de 51,3 anos, sendo $62,8 \%$ do sexo feminino e $72,6 \%$ casados. Os tipos de neoplasia mais prevalentes foram câncer de mama, pulmão e intestino. Foram alocados 57 pacientes no Grupo Experimental e 56 pacientes no Grupo Controle. Houve diminuição dos níveis de depressão e ansiedade nos pacientes do Grupo Experimental, com variação dos escores

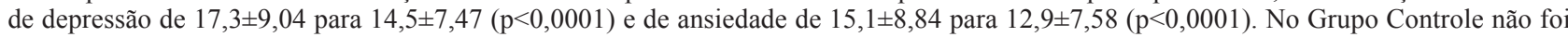
observada diferença significativa entre a avaliação inicial e final.

Conclusões: A intervenção através da técnica de relaxamento com imagem guiada mostrou eficácia em reduzir os níveis de depressão e ansiedade nessa amostra de pacientes oncológicos em vigência de quimioterapia.

DESCRITORES: terapia de relaxamento; psiconeuroimunologia; depressão; ansiedade; câncer.

\section{ABSTRACT}

Aims: To evaluate the efficacy of guided imagery relaxation in decreasing depression and anxiety levels in cancer patients submitted to chemotherapy.

Methods: A nonrandomized clinical trial was performed in male and female patients aged 30 years or older submitted to chemotherapy at a university hospital in Porto Alegre, southern Brazil. The patients were divided into an Experimental Group, which received the intervention (relaxation technique), and into a Control Group, which did not receive it. Twelve-minute guided imagery relaxation sessions were performed for 14 weeks, always during the chemotherapy procedure. In order to evaluate depression and anxiety levels, the Beck Depression Inventory and Beck Anxiety Inventory were applied at baseline and at the end of the study period. The data were analyzed by the EpiInfo 7.0 statistical software using Student's t, Wilcoxon-Mann-Whitney, and Pearson's chi-square tests. Statistical significance was obtained when $\mathrm{p}<0.05$.

Results: A total of 113 participants with a mean age of 51.3 years, among whom $62.8 \%$ were female and $72.6 \%$ were married, were included in the study. Breast, lung, and intestinal cancers were the most prevalent types of neoplasms. Fifty-seven patients were assigned to the Experimental Group and 56 to the Control Group. There was a decrease in depression and anxiety levels in the Experimental Group, in which depression scores ranged from $17.3 \pm 9.04$ to $14.5 \pm 7.47(\mathrm{p}<0.0001)$ and anxiety scores ranged from $15.1 \pm 8.84$ to $12.9 \pm 7.58(\mathrm{p}<0.0001)$. No significant difference was observed in the Control Group between the baseline and final evaluations.

Conclusions: The guided imagery relaxation technique was efficacious in reducing depression and anxiety levels in this sample of chemotherapytreated cancer patients.

KEY WORDS: relaxation therapy; psychoneuroimmunology; depression; anxiety; cancer. 
Abreviaturas: BDI, Inventário de Depressão de Beck; BAI, Inventário de Ansiedade de Beck; GE, Grupo Experimental; GC, Grupo Controle.

\section{INTRODUÇÃO}

Em pacientes oncológicos a depressão é o transtorno psiquiátrico mais comum. A prevalência de depressão nos pacientes com câncer pode variar de $22 \%$ a $29 \%$ [1]. Essa diferença de prevalência está associada ao tipo e estágio clínico do tumor, além da dor, debilidade funcional, fragilidade e suporte familiar e social que os pacientes possuem [2]. O tratamento quimioterápico está associado a prevalências ainda maiores de depressão, podendo chegar a 58\% [1]. A presença de depressão tem sido associada a um pior prognóstico e ao aumento da mortalidade pelo câncer [3].

Bottino et al. [1], em uma extensa revisão da literatura, concluíram que o estresse, a ansiedade e a depressão causam diminuição da capacidade imunológica. Sentimentos de tristeza e angústia podem fazer com que o paciente não procure ajuda, dificultando o reconhecimento da depressão, embora o tratamento com antidepressivos seja efetivo e melhore a adesão ao tratamento do câncer, reduzindo efeitos adversos [1].

As terapias complementares têm se destacado entre as opções terapêuticas em centros de tratamento de câncer nos Estados Unidos da América. A aceitação e o uso resultam em um impacto positivo imediato sobre o estresse e o sofrimento do paciente. Várias são as técnicas de intervenção utilizadas no manejo da dor e do sofrimento físico e psíquico dos pacientes oncológicos [4]. As técnicas de relaxamento são objeto de pesquisa há décadas, como instrumento importante no processo integrativo entre mente e corpo, compreendida como uma técnica complementar de intervenção junto a pacientes com doenças orgânicas [5].

Este estudo buscou avaliar a eficácia da técnica de relaxamento com imagem guiada na diminuição dos sintomas de depressão e ansiedade em pacientes oncológicos que estavam realizando tratamento quimioterápico.

\section{MÉTODOS}

Foi realizado um ensaio clínico não randomizado, cuja amostra constituiu-se de pacientes com diagnóstico de câncer em diferentes órgãos, identificados a partir da análise de prontuários. Foram incluídos pacientes com idade igual ou superior a 30 anos, com o diagnóstico estabelecido de câncer e iniciando o tratamento quimioterápico, independente do estadiamento ou plano quimioterápico (número de sessões, efeitos adversos e duração do tratamento). Os participantes foram divididos em dois grupos: Grupo Experimental (GE), que recebeu intervenção de sessões de relaxamento com imagem guiada, e Grupo Controle (GC), sem intervenção da técnica de relaxamento. A seleção dos pacientes foi feita de forma aleatória: o GE constituiu-se de pacientes do turno da manhã, enquanto o GC formou-se de pacientes que iniciavam a quimioterapia no turno da tarde. A coleta de dados foi realizada em um hospital universitário de Porto Alegre, no setor de quimioterapia. Os participantes eram encaminhados pelos oncologistas e enfermeiros à pesquisadora, que fazia o contato com o paciente, explicando os objetivos da pesquisa e posteriormente apresentando o Termo de Consentimento Livre e Esclarecido. Foram excluídos da amostra pacientes que apresentassem dificuldades cognitivas que pudessem prejudicar o entendimento das questões apresentadas nos instrumentos de avaliação.

Tanto o GE quanto o GC foram avaliados em dois momentos diferentes, que denominamos "avaliação inicial" e "avaliação final". A avaliação inicial foi realizada no momento em que era iniciado o tratamento quimioterápico e incluiu o preenchimento de uma ficha de dados demográficos e de estilo de vida (idade, sexo, estado civil, escolaridade, religião, suporte familiar, atividade física e atividades de lazer) e dados clínicos (doença e tratamento mental prévio). Após o preenchimento da ficha foram aplicadosos instrumentos de mensuração de depressão e de ansiedade, constituídos respectivamente pelo Inventário de Depressão de Beck (BDI: Beck Depression Inventory) e pelo Inventário de Ansiedade de Beck (BAI: Beck Anxiety Inventory). O BDI é uma escala de autorrelato, com 21 itens, cada um com quatro alternativas, com escores de 0 a 3 . O escore total permite classificar a depressão em níveis de intensidade. O BAI também é uma escala de autorrelato, que mede a intensidade de sintomas de ansiedade. É constituído de 21 itens que são avaliados pelo sujeito numa escala de quatro pontos que refletem os níveis de gravidade crescente de cada sintoma [6]. A avaliação final foi realizada 14 semanas após a avaliação inicial, sendo aplicados novamente os mesmos instrumentos nos dois grupos.

Durante o desenvolvimento da pesquisa, os pacientes do GE receberam uma sessão de relaxamento com imagem guiada a cada sessão de quimioterapia realizada. O relaxamento era aplicado por psicóloga apta a realizar a técnica, em ambiente terapêutico adequado, com duração de 12 minutos cada sessão, sendo 
realizado enquanto o paciente fazia a quimioterapia. As sessões de relaxamento eram conduzidas por uma gravação realizada pela pesquisadora. Solicitava-se que o paciente se posicionasse de forma confortável na poltrona e começasse a relaxar todas as partes do corpo, prestando atenção aos movimentos respiratórios. Quando o paciente demonstrava estar relaxado, solicitava-se, de forma detalhada, que o mesmo visualizasse imagens de lugares calmos (imagens de elementos da natureza). Após esse momento, o paciente era levado a imaginar-se visualizando o(s) órgão(s) em que o câncer se encontrava, bem como imaginar o medicamento quimioterápico entrando em seu corpo e destruindo as células cancerosas e, ao mesmo tempo, devolvendo saúde e equilíbrio às células saudáveis, restituindo o estado físico anterior ao câncer. Após todo esse percurso era solicitado ao paciente que retornasse lentamente ao estado em que iniciou a sessão.

Todas as sessões de relaxamento com imagem guiada foram conduzidas da mesma forma. Os pacientes do GE recebiam a intervenção a cada sessão de quimioterapia que realizavam, portanto, o número de sessões de relaxamento variou conforme o plano quimioterápico. A frequência variou entre duas e onze sessões, sendo que o número médio foi de quatro sessões considerando todos os pacientes do GE.

Os dados coletados foram tabulados utilizando o programa Microsoft Office Excel 2007 e analisados no software estatístico Epi Info 7.0. A análise descritiva contemplou medidas de frequência, tendência central e dispersão. A homogeneidade entre os grupos foi testada no que se refere à distribuição das características sociodemográficas e clínicas, bem como as médias dos testes aplicados nos grupos na avaliação inicial e final. As possíveis diferenças das médias iniciais entre os dois grupos foram testadas pelo teste $t$ de Student ou Wilcoxon-Mann-Whitney. A associação entre as medidas categóricas foi avaliada pelo teste Qui-quadrado de Pearson. Foram considerados significativos valores $\mathrm{p}<0,05$.

O estudo teve início após a aprovação do projeto pelo Comitê de Ética em Pesquisa da Pontifícia Universidade Católica do Rio Grande do Sul, sob protocolo de $\mathrm{n}^{\mathrm{O}}$ 1.172.234. Todos os participantes foram informados e consultados acerca dos procedimentos e assinaram o Termo de Consentimento Livre e Esclarecido. Foram seguidas todas as recomendações da Resolução 466/12 do Conselho Nacional de Saúde.

\section{RESULTADOS}

O estudo incluiu 113 participantes, 57 no GE e 56 no GC. A Tabela 1 apresenta os tipos de neoplasia identificados na amostra pesquisada, sendo câncer de mama, de pulmão e de intestino os mais frequentes.

Tabela 1. Localizações de câncer encontradas em uma amostra de 113 pacientes oncológicos em vigência de tratamento quimioterápico, divididos entre Grupo Experimental, que recebeu a intervenção "relaxamento com imagem guiada" $(n=57)$ e Grupo Controle $(n=56)$, que não recebeu a intervenção.

\begin{tabular}{lccc}
\hline \multicolumn{1}{c}{ Localização do câncer } & $\begin{array}{c}\text { Grupo Experimental } \\
\mathbf{n}(\mathbf{\%})\end{array}$ & $\begin{array}{c}\text { Grupo Controle } \\
\mathbf{n}(\%)\end{array}$ & $\begin{array}{c}\text { Total } \\
\mathbf{n}(\%)\end{array}$ \\
\hline Mama & $9(15,78)$ & $13(23,21)$ & $22(19,46)$ \\
Pulmão & $9(15,78)$ & $11(19,64)$ & $20(17,69)$ \\
Intestino/reto & $7(12,28)$ & $6(10,71)$ & $13(11,5)$ \\
Ossos & $6(10,52)$ & $3(5,35)$ & $9(7,96)$ \\
Próstata & $3(5,26)$ & $3(5,35)$ & $6(5,3)$ \\
Linfoma Hodgkin / ñ Hodgkin & $0(0,00)$ & $5(8,92)$ & $5(4,42)$ \\
Colo do útero & $4(7,01)$ & $1(1,78)$ & $5(4,42)$ \\
Ovário & $3(5,26)$ & $1(1,78)$ & $4(3,53)$ \\
Fígado & $2(3,5)$ & $1(1,78)$ & $3(2,65)$ \\
Cérebro & $2(3,5)$ & $1(1,78)$ & $3(2,65)$ \\
Estômago & $2(3,5)$ & $1(1,78)$ & $3(2,65)$ \\
Testículo & $2(3,5)$ & $0(0,00)$ & $2(1,76)$ \\
Pâncreas & $1(1,75)$ & $1(1,78)$ & $2(1,76)$ \\
Melanoma/pele & $0(0,00)$ & $2(3,57)$ & $2(1,76)$ \\
Outros* & $7(12,28)$ & $7(12,5)$ & $14(12,38)$ \\
Total & $57(100)$ & $56(100)$ & $113(100)$ \\
\hline
\end{tabular}

* rim (2); sarcoma de partes moles (2); sarcoma de Ewing (2); liposarcoma de mediastino (2); pênis (2); glioma (1); orofaringe (1); esôfago (1); leucemia(1). 
A média de idade dos pacientes foi de 51,3 anos, $62,8 \%$ eram do sexo feminino e $72,6 \%$ eram casados. A maioria $(94,7 \%)$ possuía religião, com predominância de católicos. Em relação à prática de atividade física e de lazer, a maioria $(81,4 \%)$ não praticava, mesmo antes de iniciar a quimioterapia. Nesses dados não houve diferença significativa entre os grupos. Quanto ao grau de instrução, 59,3\% possuiam ensino fundamental e uma minoria possuía ensino superior. Apenas $8 \%$ da amostra estavam estudando. Observamos diferenças estatisticamete significativas no grau de instrução entre os dois grupos, $\mathrm{p}=0,0052$. No GE $68,4 \%$ dos participantes tinha ensino fundamental, contra $50 \%$ no GC (Tabela 2).

Dos 113 participantes $104(92,0 \%)$ sentia-se apoiada e acolhida pela família durante o diagnós- tico e tratamento do câncer, $51(91,0 \%)$ do GC e $53(92,9 \%)$ do GE. A variável não obteve diferença estatisticamente significativa entre os grupos. Também não houve diferença estatisticamente significativa entre os grupos quanto ao fato de terem apresentado doença mental anterior ao diagnóstico de câncer. Conforme os resultados, $42 \%$ da amostra tinha desenvolvido algum transtorno mental antes do diagnóstico de câncer. Quando questionados se haviam buscado auxílio psicológico ou psiquiátrico, $68,1 \%$ afirmaram que nunca haviam procurado por atendimento na área de saúde mental. Os pacientes GC e GE mostraram homogeneidade quanto à variável atendimento psicológico e/ou psiquiátrico, $\mathrm{p}=0,0832$ (Tabela 2).

Tabela 2. Distribuição das características sociodemográficas e clínicas da amostra total, do Grupo Experimental, que recebeu a intervenção "relaxamento com imagem guiada", e do Grupo Controle, que não recebeu a intervenção.

\begin{tabular}{|c|c|c|c|c|}
\hline \multirow[t]{2}{*}{ Variável } & $\begin{array}{l}\text { Amostra total } \\
n=113\end{array}$ & $\begin{array}{l}\text { Grupo Experimental } \\
n=57\end{array}$ & $\begin{array}{l}\text { Grupo Controle } \\
n=56\end{array}$ & \multirow{2}{*}{$\mathbf{p}$} \\
\hline & n (\%) & n (\%) & n (\%) & \\
\hline $\begin{array}{l}\text { Gênero } \\
\text { Feminino } \\
\text { Idade (média em anos) }\end{array}$ & $\begin{array}{c}71(62,8) \\
51,3\end{array}$ & $\begin{array}{l}35(61,40) \\
52,08\end{array}$ & $\begin{array}{l}36(64,29) \\
50,62\end{array}$ & 0,7512 \\
\hline $\begin{array}{l}\text { Estado Civil } \\
\text { Casado } \\
\text { Solteiro } \\
\text { Separado/divorciado } \\
\text { Viúvo }\end{array}$ & $\begin{array}{l}82(72,6) \\
11(9,7) \\
11(9,7) \\
9(8,0)\end{array}$ & $\begin{array}{l}42(73,68) \\
7(12,28) \\
5(8,77) \\
3(5,26)\end{array}$ & $\begin{array}{c}40(71,43) \\
4(7,14) \\
6(10,71) \\
6(10,71)\end{array}$ & 0,5830 \\
\hline $\begin{array}{l}\text { Atividade física } \\
\text { Não pratica }\end{array}$ & $92(81,4)$ & $47(82,46)$ & $45(80,36)$ & 0,7742 \\
\hline $\begin{array}{l}\text { Atividade de Lazer } \\
\text { Não realiza }\end{array}$ & $78(69,0)$ & $38(66,67)$ & $40(71,43)$ & 0,5841 \\
\hline $\begin{array}{l}\text { Está estudando } \\
\text { Sim }\end{array}$ & $9(8,0)$ & $5(8,77)$ & $4(7,14)$ & 0,5112 \\
\hline $\begin{array}{l}\text { Grau de instrução } \\
\text { Ensino fundamental } \\
\text { Ensino médio } \\
\text { Ensino superior }\end{array}$ & $\begin{array}{l}67(59,3) \\
35(31,0) \\
11(9,70)\end{array}$ & $\begin{array}{l}39(68,42) \\
10(17,54) \\
8(14,04)\end{array}$ & $\begin{array}{c}28(50,0) \\
25(44,64) \\
3(5,36)\end{array}$ & 0,0052 \\
\hline $\begin{array}{l}\text { Possui religião } \\
\text { Sim } \\
\text { - Católica } \\
\text { - Evangélica } \\
\text { - Espírita } \\
\text { - Luterana } \\
\text { - Outras }\end{array}$ & $\begin{array}{c}107(94,7) \\
60(56,0) \\
28(26,2) \\
14(13,1) \\
3(2,8) \\
2(1,9)\end{array}$ & $\begin{array}{c}52(91,23) \\
28(53,85) \\
16(30,77) \\
5(9,62) \\
1(1,92) \\
2(3,85)\end{array}$ & $\begin{array}{c}55(98,21) \\
32(58,18) \\
13(23,64) \\
9(16,36) \\
1(1,82) \\
0(0,00)\end{array}$ & 0,2061 \\
\hline $\begin{array}{l}\text { Apoio familiar } \\
\text { Sim }\end{array}$ & $104(92,0)$ & $53(92,98)$ & $51(91,07)$ & 0,7424 \\
\hline $\begin{array}{l}\text { Doença mental ao longo da vida } \\
\text { Sim }\end{array}$ & $47(42,0)$ & $20(35,09)$ & $27(48,21)$ & 0,1569 \\
\hline $\begin{array}{l}\text { Transtorno mental antes do câncer } \\
\text { Transtorno depressivo } \\
\text { Transtorno de ansiedade } \\
\text { Transtorno bipolar } \\
\text { Estresse pós-traumático }\end{array}$ & $\begin{array}{c}34(72,3) \\
8(17,0) \\
3(6,4) \\
2(4,2)\end{array}$ & $\begin{aligned} 11 & (19,30) \\
5 & (8,77) \\
2 & (3,51) \\
2 & (3,51)\end{aligned}$ & $\begin{aligned} 23 & (41,07) \\
3 & (5,36) \\
1 & (1,79) \\
0 & (0,00)\end{aligned}$ & 0,0905 \\
\hline $\begin{array}{l}\text { Realização de tratamento psicológico/psiquiátrico } \\
\text { Nunca realizou } \\
\text { Realizou no passado } \\
\text { Realiza atualmente }\end{array}$ & $\begin{array}{l}77(68,1) \\
24(21,2) \\
12(10,6)\end{array}$ & $\begin{array}{c}45(78,94) \\
8(14,03) \\
4(7,01)\end{array}$ & $\begin{array}{l}32(57,14) \\
16(28,57) \\
8(14,29)\end{array}$ & 0,0832 \\
\hline
\end{tabular}


Entre os participantes que mencionaram ter algum transtorno psiquiátrico anterior ao diagnóstico de câncer, a maioria $(72,3 \%)$ relatou quadros de transtorno depressivo. Ao analisarmos os tipos de doença mental desenvolvidos anteriormente pelos participantes, percebemos que não houve diferença estatisticamente significativa entre os grupos, $p=0,090$. Entretanto no GC houve um número maior de participantes com transtorno depressivo anterior ao diagnótico de câncer do que no GE. Em contrapartida, no GE houve um número maior de participantes com transtorno de ansiedade. Um pequeno número mencionou transtorno bipolar e transtorno de estresse pós-traumático ao longo da vida (Tabela 2).

A Tabela 3 mostra os resultados dos testes aplicados para avaliar os níveis de depressão e ansiedade nos participantes, comparando a avaliação inicial com a final. Quanto aos níveis de depressão, na avaliação inicial do GE, os pacientes apresentavam uma média de 17,3 e na avaliação final esta média foi de 14,5 , apontando uma redução da depressão, embora o escore médio do BDI tenha permanecido no nível leve de depressão. Assim, comparando o BDI inicial com o BDI final o GE teve uma redução média significativa de $2,7 \pm 4,43(p<0,001)$. Já o GC a média inicial do BDI foi de 20,02 e a final foi de 20,5, representando um aumento médio não significativo de $0,5 \pm 4,34$ no escore do BDI.

Em relação aos níveis de ansiedade, na avaliação inicialdo GE a média do escore do BAI foi de $15,07 \pm 8,84$, enquanto que na avaliação final a média diminuiu para $12,9 \pm 7,58$. Assim, houve uma diminuição no escore médio do GE de 2,19, com diferença significativa ( $p<0,001$ ), enquanto que no GC a média do BAI aumentou não significativamente ao longo do período de pesquisa.

\section{DISCUSSÃO}

Este estudo analisou a eficácia da técnica de relaxamento com imagem guiada na redução de sintomas de ansiedade e depressão, com a intenção de minimizar o sofrimento do paciente oncológico e proporcionar-lhe melhor qualidade de vida. Além dos tratamentos farmacológicos, a literatura tem demonstrado que as técnicas de relaxamento oferecem um efeito positivo em certas condições médicas, tais como dor e depressão [7].

A única variável sociodemográfica que mostrou diferença entre os grupos foi o grau de instrução. Acredita-se que essa diferença de escolarização entre os grupos não tenha interferido nos resultados obtidos, uma vez que a pesquisadora aplicava os testes de forma individual, lendo em voz alta as questões e acompanhando a compreensão pelo pesquisado. Quanto às atividades físicas e de lazer, observou-se que a maioria dos participantes não as praticava, embora a atividade física regular seja recomendada para prevenção e tratamento de hipertensão, diabetes não insulinodependente, dislipidemia, obesidade, doenças crônicas como osteoporose e alguns tipos de câncer, redução do estresse e melhora da autoestima [8]. O diagnóstico de câncer e seu tratamento são situações que afetam a saúde psicológica dos pacientes, especialmente nos casos de cirurgias que envolvem mutilação e diminuição da capacidade de executar tarefas, repercutindo na qualidade de vida, no nível de atividade física e na autoestima [9]. O fato de estarem realizando tratamento quimioterápico, que envolvia efeitos colaterais: desânimo, fraqueza, cansaço, náuseas e tonturas e edemas em membros superiores poderia explicar a não realização de atividade física, embora a maioria dos pacientes também tenha relatado

Tabela 3. Resultados dos testes Inventário de Depressão de Beck (BDI) e Inventário de Ansiedade de Beck (BAI) em uma amostra de 113 pacientes, divididos entre Grupo Experimental, que recebeu a intervenção "relaxamento com imagem guiada" $(n=57)$ e Grupo Controle $(n=56)$, que não recebeu a intervenção.

\begin{tabular}{|c|c|c|c|c|c|}
\hline Variável & Grupo Experimental* & $p^{+}$ & Grupo Controle* & $p^{+}$ & $p^{\ddagger}$ \\
\hline BDI inicial & $17,26 \pm 9,04$ & & $20,0 \pm 7,82$ & & 0,0861 \\
\hline BDI final & $14,53 \pm 7,47$ & & $20,5 \pm 8,22$ & & 0,0001 \\
\hline BDI diferença & $-2,74 \pm 4,43$ & $<0,0001$ & $0,48 \pm 4,34$ & 0,7971 & 0,0001 \\
\hline BAI inicial & $15,1 \pm 8,84$ & & $15,9 \pm 7,24$ & & 0,5738 \\
\hline BAI final & $12,9 \pm 7,58$ & & $16,4 \pm 6,85$ & & 0,0106 \\
\hline BAI diferença & $-2,19 \pm 4,12$ & $<0,0001$ & $0,48 \pm 3,50$ & 0,8487 & 0,0003 \\
\hline
\end{tabular}

BDI, Inventário de Depressão de Beck; BAI, Inventário de Ansiedade de Beck.

* Média \pm desvio padrão.

† Comparação entre as médias iniciais e finais.

₹ Comparação entre os grupos. 
a ausência desse hábito previamente ao início da quimioterapia.

Observou-se que a grande maioria dos pacientes estudados sentia-se apoiada pela família. Quando o paciente recebe apoio da família os impactos da doença podem ser minimizados. Dessa forma, é importante que a equipe profissional procure apoiar a família, para que esta por sua vez tenha condições de dar o apoio necessário ao paciente no enfrentamento da doença $[10,11]$. A doença oncológica acarreta problemas físicos, psicológicos, sociais e financeiros, influenciando na atuação social e familiar, trazendo consequências para a qualidade de vida do paciente $\mathrm{e}$ de sua família [12].

Outro aspecto investigado neste estudo foi a presença ou não de doenças psiquiátricas anteriores ao diagnóstico de câncer e se as mesmas influenciaram nos resultados. Estudos apontam possíveis relações entre quadros psiquiátricos e desenvolvimento de doenças crônicas como o câncer, mas ainda de forma especulativa. A cronificação de determinados quadros deterioraria a função imune e a vigilância do sistema imunológico em relação às células cancerosas [13]. Neste estudo não houve diferença estatisticamente significativa entre os grupos quanto ao fato de terem apresentado doença mental anterior ao diagnóstico de câncer. Apenas uma minoria havia desenvolvido algum transtorno mental antes do diagnóstico de câncer e, entre estes, a maioria foi diagnosticada com transtorno depressivo. A análise da modulação psicológica do sistema imune sugere que os quadros depressivos aumentam os níveis de citocinas pró-inflamatórias, especialmente a interleucina 6 , o que justificaria as comorbidades clínicas associadas a esses quadros. Ocorre também a redução da atividade das células Natural Killer e de contagens de linfócitos T [14].

Um estudo realizado em mulheres com câncer de mama constatou a atuação de fatores emocionais no surgimento de neoplasias. Os estados afetivos, particularmente a depressão, relacionam-se ao aparecimento do câncer, dada sua influência no sistema imunológico. A forma como o indivíduo avalia, enfrenta e supera as situações de estresse mantém relação com a diminuição do funcionamento do sistema imunológico e o adoecimento $[15,16]$.

Além disso, ao receber o diagnóstico de câncer, a integridade psicológica do paciente fica fragilizada e problemas de ordem emocional ocorrem frequentemente, tornando-o vulnerável a transtornos depressivos e ansiosos. Surgem sentimentos de angústia e medo da mutilação, da dor e da morte [10]. Esses pacientes podem também apresentar outros sintomas, como o medo da recidiva, fadiga, problemas de sono, alterações na imagem corporal, disfunção sexual, ansiedades relacionadas ao tratamento e problemas de relacionamento conjugal [13]. Os resultados encontrados neste estudo estão alinhados com estudos publicados acerca da eficácia das técnicas de relaxamento na redução de sintomas depressivos e estresse em pacientes com câncer. Além disso outras técnicas psicossociais, terapia individual e em grupo, também são descritas na literatura com bons resultados [1].

Um ensaio clínico randomizado foi realizado com 34 mulheres portadoras de câncer de mama em tratamento radioterápico. Após as intervenções de sessão de relaxamento com imagem guiada houve melhora significativa nos índices de estresse e nos níveis de depressão e ansiedade das pacientes, porém não houve alteração dos níveis de cortisol ou proliferação celular [17]. Outro estudo similar, realizado no Hospital das Clínicas de São Paulo, avaliou o efeito da intervenção de relaxamento com imagem guiada sobre a qualidade de vida relacionada à saúde e a depressão em pacientes com câncer durante tratamento quimioterápico. Para os pesquisadores, a intervenção de relaxamento com imagem guiada melhorou a qualidade de vida relacionada à saúde em várias funções e também diminuiu os níveis de depressão [2]. Esses dois estudos estão de acordo com os resultados encontrados nesta pesquisa, de que a técnica de relaxamento com imagem guiada auxilia na diminuição dos níveis de depressão e ansiedade em pacientes oncológicos em tratamento.

Uma revisão de literatura investigou os efeitos de técnicas complementares no controle de ansiedade, dor, fadiga e sono em oncologia. Os estudos revisados demonstraram a eficácia das técnicas complementares, além de vantagens como o baixo custo e a facilidade de aplicação, dispensando recursos tecnológicos [4]. Pesquisadores chineses também investigaram os efeitos do relaxamento e da musicoterapia em 170 mulheres mastectomizadas. O estudo, um ensaio clínico randomizado e controlado, mostrou que no grupo de intervenção foram reduzidos a depressão, a ansiedade e o tempo de permanência hospitalar em relação ao grupo controle [18]. Os procedimentos mais utilizados nas pesquisas têm sido distração cognitivo/atencional, hipnose/imagem guiada, dessensibilização sistemática, reestruturação cognitiva e treinos de relaxamento [19].

Os resultados do presente estudo permitem concluir que a intervenção através da técnica de relaxamento com imagem guiada mostrou-se eficaz nesta amostra de pacientes oncológicos em vigência de 
quimioterapia, pois os resultados dos instrumentos BDI e BAI evidenciaram redução nos níveis de depressão e ansiedade no grupo que recebeu a intervenção.

Com base em evidências publicadas anteriormente, podemos dizer que a técnica de relaxamento, auxiliando na redução dos níveis de estresse, depressão e ansiedade, pode oferecer melhora na qualidade de vida e estimular o fortalecimento do sistema imunológico. Neste sentido é importante que o psicólogo e os demais profissionais da área da saúde compreendam a interação corpo-mente, compreensão esta que é importante para que o profissional tenha capacidade de auxiliar o paciente no reestabelecimento da sua saúde.

Esta pesquisa não pretendeu esgotar o assunto, mas suscitar mais estudos na área da psico-oncologia e da psiconeuroimunologia. Salientamos a importância do desenvolvimento de pesquisas relacionadas à utilização das técnicas de relaxamento como recurso complementar na promoção e tratamento da saúde, principalmente em pacientes com câncer e outras doenças crônicas.

\section{NOTAS}

\section{Apoio financeiro}

Este estudo recebeu apoio financeiro da Coordenação de Aperfeiçoamento de Pessoal de Nível Superior (CAPES) por meio do Programa de Suporte à Pós-Graduação de Instituições de Ensino Particulares (PROSUP).

\section{Declaração de conflito de interesses}

Os autores declaram não haver conflitos de interesses relevantes ao conteúdo deste estudo, informam ter tido acesso a todos os dados obtidos e assumem completa responsabilidade pela integridade dos resultados.

\section{REFERÊNCIAS}

1. Bottino SMB, Fráguas R, Gattaz WF. Depressão e câncer. Rev Psiquiatr Clín. 2009;36 Suppl 3:109-15. https://doi.org/10.1590/S010160832009000900007

2. Nicolussi AC. Relaxamento com imagem guiada: influência sobre a qualidade de vida relacionada à saúde de pacientes com câncer durante o tratamento quimioterápico [tese]. Ribeirão Preto: Escola de Enfermagem de Ribeirão Preto; 2012.

3. Wong E, Rowbottom L, Tsao M, Zhang L, McDonald R, Danjoux C, Barnes E, Chan S, Chow E. Correlating symptoms and their changes with survival in patients with brain metastases. Ann Palliat Med. 2016;5(4):253-66. https://doi.org/10.21037/apm.2016.09.01

4. Costa AIS, Reis PED. Técnicas complementares para controle de sintomas oncológicos. Rev Dor. 2014;15(1):61-4.

5. Elias ACA, Giglio JS, Pimenta CAM, El-Dash LG. Programa de treinamento sobre a intervenção terapêutica "relaxamento, imagens mentais e espiritualidade" (RIME) para re-significar a dor espiritual de pacientes terminais. Rev Psiquiatr Clín. 2007;34 Suppl 1:6072. https://doi.org/10.1590/S0101-60832007000700009

6. Cunha JÁ, organizador. Manual da versão em português das Escalas Beck.São Paulo: Casa do Psicólogo; 2001.

7. Gardiner P, Mitchell S, Filippelli AC, Sadikova E, White LF, Paasche-Orlow MK, Jack BW. Health literacy and complementary and alternative medicine use among underserved inpatients in a safety net hospital. J Health Commun. 2013;18 Suppl 1:290-7. https:/ doi.org/10.1080/10810730.2013.830663

8. Sabino Neto M, Moreira JR, Resende V, Ferreira LM. Nível de atividade física em mulheres mastectomizadas e submetidas a reconstrução mamária. Rev Bras Cir Plást. 2012;27(4):556-61. https://doi.org/10.1590/S1983-51752012000400015

9. Rivera HR. Depression symptoms in cancer caregivers. Clin J Oncol Nurs. 2009;13(2):195-202. https://doi.org/10.1188/09.CJON. 195-202

10. Ceolin VES. A família frente ao diagnóstico do câncer. In: Hart CFM, organizador. Câncer: uma abordagem psicológica. Porto Alegre: AGE; 2008. p. 118-28.

11. Bellato R, Araújo LFS, Dolina JV, Musquim CA, Corrêa GHLST. Experiência familiar de cuidado na situação crônica. Rev Esc Enferm USP. 2016;50(n. esp):81-8.

12. Odgen JE. Psicologia da saúde. 2. ed. Lisboa: Climepsi; 2004.

13. Cantinelli FS, Camacho RS, Smaletz O, Gonsales BK, Braguittoni E, Rennó Jr J. A oncopsiquiatria no câncer de mama: considerações a respeito de questões do feminino. Rev Psiquiatr Clín. 2006;33(3):124-33. https://doi.org/10.1590/S0101-60832006000300002

14. Kiecolt-Glaser JK, McGuire L, Robles TF, Glaser R. Psychoneuroimmunology and psychosomatic medicine: back to the future. Psychosom Med. 2002;64(1):15-28. https://doi.org/10.1097/00006842-200201000-00004

15. Neme CMB, Lipp MEN. Estresse psicológico e enfrentamento em mulheres com e sem câncer. Psic Teor e Pesq. 2010;26(3):475-83. https://doi.org/10.1590/S0102-37722010000300010

16. Lipp MEN. Stress e o turbilhão da raiva. São Paulo: Casa do Psicólogo; 2005. 
17. Nunes DF, Rodriguez AL, da Silva Hoffmann F, Luz C, Braga Filho AP, Muller MC, Bauer ME. Relaxation and guided imagery program in patients with breast cancer undergoing radiotherapy is not associated with neuroimmunomodulatory effects. J Psychosom Res. 2007;63(6):647-55. https://doi.org/10.1016/j.jpsychores.2007.07.004

18. Zhou K, Li X, Li J, Liu M, Dang S, Wang D, Xin X. A clinical randomized controlled trial of music therapy and progressive muscle relaxation training in female breast cancer patients after radical mastectomy: results on depression, anxiety and length of hospital stay. Eur J Oncol Nurs. 2015;19(1):54-9. https://doi.org/10.1016/j.ejon.2014.07.010

19. Lopes RFF, Santos MR, Lopes EJ. Efeitos do relaxamento sobre a ansiedade e desesperança em mulheres com câncer. Rev Bras Ter Comport Cogn. 2008;10(1):39-49. 